\title{
Electrophysiological Modeling In Generalized Epilepsy Using Surface EEG And Anatomical Brain Structures
}

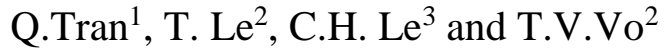 \\ ${ }^{1}$ Pham Ngoc Thach Medical University, Ho Chi Minh City, Vietnam \\ ${ }^{2}$ Biomedical Engineering Department, International University-Vietnam National University, Ho Chi Minh City, Vietnam \\ ${ }^{3}$ Faculty of Engineering and Science, University of Greenwich, Kent, United Kingdom
}

\begin{abstract}
Deep brain structures involve significantly in the pathology of brain diseases such as epilepsy, Alzheimer,and Parkinson. Physiological brain modeling has become an emerging approach to investigatethe coupling dynamics of the brain activity ofthese diseases. We propose a method using the surface EEG signals integrated with the anatomical individual brain to build the electrophysiological model of the epileptic patient's brain. The EEG-driven model is used to investigate the deep brain activities of 23 patients diagnosed with generalized epilepsy fromCHB-MIT Scalp EEG Database. Significant changesin the electrical activities in hippocampus, accumbens, amygdala, provide us insights into the dynamics ofactive brain regions during epilepsy. All of these brain regions show the significant energy variation defined by 5 features (Mean, Max, Min, Standard deviation, Power spectral density) with the p-value $<0.05$ in both pre-ictal vs ictal and ictal vs post-ictal. Such result shows the potential of using EEG as a tool to capture the deep brain activity of epilepsy andother diseases that alter deep brain structures. The proposed model may be used to enhance the sensitivity of detecting and predicting epilepsy, detect the progression of the brain lesion, and support the decision-making for a brain medical intervention.
\end{abstract}

Keywords- Electrophysiological model, generalized epilepsy, deep brain structures, deep brain activity.

\section{INTRODUCTION}

Epilepsy is the most common neurological disorder that isdiagnosed in about50 million people worldwide[1]. The pathology of epilepsy involves the abnormal neuronal discharge that causes the disturbance in brain function called aseizure. In particular, epilepsy causes recurrent seizures accompanying with losing consciousness in some severe cases. These seizures can be dangerous due to its almost unpredicted occurrence. Thus, the patients' quality of life is decreased as their activity are limited from joining traffic or doing recreation. Besides, sudden unexplained death in epilepsy (SUDEP) can occur in a small percentage of patient[2], which can be a formidable happening. Although seizures occurrence can be managed by anticonvulsant drug or surgery procedure, the seizures can only reduce but not treated completely. There are about $22 \%$ of patients considered to have good postsurgical outcomes, despite having simple partial seizures and $31 \%$ of all operated patients had a complex pattern of remission and relapse [3]. Getting to know the nature of brain activity during epilepsy will spark new medical interventions that completely eradicate the disease.

The interactions between different regions of the brain during a seizurefrom functional and structuralaspects may spark the idea of how epilepsy starts at the first place [4]. While the cerebral cortex roles related to areas on the surface of the brain of epilepsy is clearly recognised, there is less conclusive data available regarding the role of subcortical structuresin human epilepsy. However, the accumulative evidenceshows that the subcortical structures such as thethalamus, basal ganglia, hypothalamus, cerebellum, and brainstem also take part in behavioral manifestations, initiation, and propagation of epileptic seizures [5]. Others studies also emphasize the role of the cortical-subcortical interaction in seizure generation [6]. Therefore, by focusing on analyzing the deep brain activity in epilepsy patient brain, we maybetter understand the original sources and the mechanism of epileptic seizures.

Previous studies have usedEEG related features todetect the abnormality of deep brain activity. In the study of anatomical and electrophysiological model of deep brain structures to evaluate the respective sensitivity of the MEG and EEG to signals from deeper origins, the results indicate that MEG/EEG can be used to localize these deeper generators[7]. A few studies use the integrated method of simultaneous EEGs and fMRI recordings in human subjects during a resting state and seizure state. The findings suggest that different subcortical structures contribute to slow and fast modulations of alpha spectral on EEG [8].The study of EEG in Parkinson disease comes to the conclusion that scalp EEG analysis can be used to detect the abnormalities in the function of the basal ganglia - cortical circuit in Parkinson patient[9]. In short, given a right approach, surface EEG can be a powerful biomarker to capture the disorders related to subcortical brain alterations.

The electrophysiological model has been widely used to explore the dynamic of epilepsy. The temporal lobe epilepsy (TLE) group used intracerebral EEG and a physiological-relevant neuronal population model to investigate the transition from interictal to ictal in human.They found that there has been an increase of excitation during aseizure preonset, an abrupt drop of dendritic inhibition at the seizure onset, and a crucial role of perisomatically projecting interneurons in observed EEG gamma and theta ictal activity during the seizure [10]. In another study, TLE utilized a macroscopic physiological model of the EEG to investigate the causality of thefunctional couplings between cerebral structures, that 
establish during seizures interpreted [11]. Localizing the source of epilepsy for pre-surgery assessment is also an interesting use of theelectrophysiological model. In another study, to localize the cortical source dynamics and interactions during aseizure,the author uses a combination method of multivariate Granger-causal and graph-theoretic metrics combined with distributed source localization by Sparse Bayesian Learning to reveal and visualize distinct seizure stage-dependent shifts in the spatiotemporal dynamics of the cortical structures [7].

We propose a method to utilize the electrophysiologicalinverse modelintegrated with the anatomical individual brainto explore the coupling dynamics of generalized epilepsy in the deep brain structures. Our electrophysiological model requiresthesurface noninvasive EEG signals as the input.To explore the activity of deep brain structures, our research involves in estimating the current density based on the EEG recordsand the anatomical structure of the brain based on the fMRI scan. The outputs - action potential energy of the brain areas-generated from the model are used to characterize brain electric activity in the subcortical structures, specify the dynamic couplings between deep cerebral areas and visualize the overall of the brain structure during an epilepsy episode. The rest of the paper is organized as follows. Section II describes the data and modeling method. The results and discussions are shown in section III. Finally, a brief conclusion is presented in Section IV.

\section{Methods}

\section{A. Data description and preprocessing}

All the surface EEG data used in this study were collected from the online CHB-MIT Scalp EEG Database, www.physionet.org. The database consists of continuous scalp EEG recordings from pediatric patients (age < 18) undergoing medication withdrawal for epilepsy surgery evaluation at Children's Hospital Boston. Data was grouped into 24 cases corresponding to 23 patients (the first and the $24^{\text {th }}$ case are from one person). The data was sampled at $256 \mathrm{~Hz}$ and recorded using the international 1020 EEG electrode configuration. In sum, there are 944 hours of continuously recorded EEG signalswith163 seizures

To investigate the anatomical originals of the seizure, we implemented a brain electrical source simulation model to characterize the electrophysiological activities of the deep brain activity during epilepsy[12]. The simulation model consists of a)aprecise head model of the patients derived from fMRI scan $b$ ) the specified set of locations for the setup of EEG leads c) 17 monopolar channels of EEG signals consisting of thepre-ictal, ictal, and post-ictal period. We utilized the 23 bipolar EEGdata from Physionet and converted it into the 17 monopolar configurations (Fig.1-a). The outputs from the model are the time series of the electrical energy from 15 deep brain structures(Fig.1-a) with each structure consist of a set of predefined voxels. The nature of these voxels are the current dipoles of deep brain structures. The color alteration these voxels indicates the changing of energy in subcortical structure denoted in the blue dotted area (Fig.1-c). The 15 deep brain areas include-2 sides of Thalamus (left and right), 2 sides of Pallidum, 2 sides of Caudate, 2 sides of Accumbens, 2 sides of Putamen, 2 sides of Hippocampus, 2 sides of Amygdala, and theBrainstem (Fig.1-b). To elaborate the steady characteristics of typical seizure episodes- normally last for 1-2 mins- each simulated epoch consists of 5 minute long of pre-ictal, full ictal period, and 5 minutes of post-ictal. In total, 180 epochs of all seizure episodes from 23 patients have been used for the brain electrical source analysis.

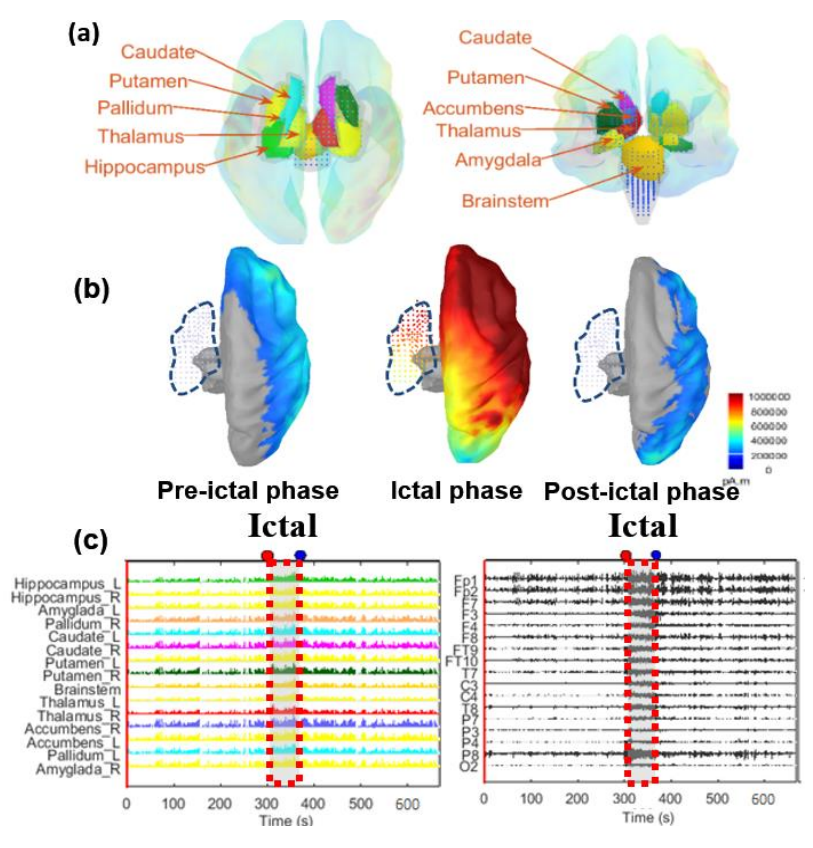

Fig. 1: (a) All 15 regions of the deep brain structures (top\& front view): 2 Hippocampus, 2 Thalamus, 2 Caudate, 2 Putamen, 2 Pallidum, 2 Amygdala, 2 Accumbens, and Brainstem. (b) The model simulation images of each phase, the color presents the electrical energy of specific brain regions, each point in the blue dotted area represents for a voxel; andthe magnitude of electrical energy are color coded. (c) The time series of the electrical energy in 15 deep brain areas derived from the model and the corresponding 17 monopolar channels of EEG consisting of $5 \mathrm{~min}$ of the pre-ictal period, ictal period, and5 min of the post-ictal period.

The features extracted from the signals of 15 deep brain areas are used to evaluate the electrophysiological variation during the epileptic seizure. To investigate the temporal characteristics along with spatial activity we used sliding window feature extraction technique. A set of 5 features including average, max, min, standard deviation and power spectral density are extracted in a fixed 5-second length sliding window. The extracted features in each window manifest the local dominant brain regions during an epilepsy episode. Besides, by analyzing the variations of the features we want to see if there are any differences in brain activity among pre-ictal, ictal, and post- ictal phase. It also helps us to know if generalized epilepsy affects the brain uniformly or selectively and the time distribution of the active deep brain regions in the epilepsy period. 


\section{B. The wMNEmodeling method}

The measured EEG signals and underlying current source strengths are related by a linear transformation:

$m(t)=\left[\begin{array}{c}m_{1}(t) \\ m_{2}(t) \\ \cdot \\ \cdot \\ m_{M}(t)\end{array}\right]=A\left[\begin{array}{c}s_{1}(t) \\ s_{2}(t) \\ \cdot \\ \cdot \\ s_{N}(t)\end{array}\right]+\boldsymbol{n}(t)=A \boldsymbol{s}(t)+\boldsymbol{n}(t)$

where $\boldsymbol{m}(t)$ stands for the measurement from $\mathrm{M}$ channels of EEG signal over the time $t, \boldsymbol{s}(t)$ is time series of the electrical power of $\mathrm{N}$ sourcedipoles, $\boldsymbol{n}(t)$ is the noise, and $A$ is the lead fields (or gain matrix) of the dipolar sources forthe given $\mathrm{M}$-channel EEG configuration. The gain matrix $A$ represents for the mapping from the current elements to the EEG sensors and can be calculated bysolving the forward model using Boundary element method (BEM)[13]. Acontains the information of each dipole such as: location, orientation, the conductivity of the field defined distinctively for each brain structure. Because $A$ is an uninvertible matrix sincethe dipoles outnumber the sensors $(N \gg M)$, source time series $\boldsymbol{s}(t)$ can not be calculated directly from the equation (1). This leads to the ill-posed problem in EEG inverse problem that the solution for $\boldsymbol{s}(t)$ is non-unique. To deal with it, the source time series can be estimated by the equation:

$\hat{\boldsymbol{s}}(t)=W \cdot \boldsymbol{m}(t)$

where $\hat{\boldsymbol{s}}(t)$ is the estimated source time series from the measured $\operatorname{EEG} \boldsymbol{m}(t)$. In MNE method, matrix $W$ can be calculateas

$W=R A^{\prime}\left(A R A^{\prime}+\lambda^{2} C\right)^{-1}$

where $R$ is the covariance matrix of the source $\boldsymbol{s}(t)$, representing the "a priori" source covariance matrix and is used to "inform" or "re-weight" the solution. $C$ is the covariance matrix of the channel noise $\boldsymbol{n}(t)$ in equation (1) and $\lambda^{2}$ is aregularization parameter to avoid magnification of errors in data in the current solution.

However, the source estimation derived from MNE can be biased toward superficial sources, because the sensitivity of the sensors decreases as thedistance from the source increases[14]. The problem can be solved by applying depth weighting[15], thus, the weighted MNE (wMNE) provides us a more precise source estimation result. The depth weight can be added to MNE by regulating $R$ in equation (3):

$$
R=\left(D^{\prime} D\right)^{-1}
$$

where $\mathrm{D}$ is the $3 \mathrm{~N} \times 3 \mathrm{~N}$ ( $\mathrm{N}$ is the number of dipoles as mentioned) weighting matrix[15]. The wMNE optimizationelectrophysiological model is implemented using Brainstorm open-source [12] and Matlab ${ }^{\text {TM }}$ software.

\section{RESULTS AND DISSCUSIONS}

The electrophysiological model can provide us the visual source estimation, and overall give us the idea of how brain activity changes during epilepsy. Fig. 2 shows the alternationsin deep brain activity fromthepre-ictal, ictal to thepost-ictalphase of an individual seizure episode. The dominant areas can be inspected in the ictal phase. From the observation, many subcortical structures involve in generalized epilepsy represents the highest activity in that certain time. Besides, there is hardly any activity recorded in the pre-ictal and post-ictal phase. The histograms of the five features in the most active brain regions over three periods (pre-ictal, ictal and post-ictal)are also generated and studied in the Fig. 3 . It is shown that not all regions of the brain are affected equally bygeneralized epilepsy. We performed the onesample Kolmogorov-Smirnov test, on each deep brain region for 3 epilepsy phases with respect to 5 functions (the total of $15 \times 3 \times 5=225$ tests), in order to test the normality of the features. We performedthe pairwise t-test to evaluatethe significant differences in the brain activity among pre-ictal and ictal, pre-ictal and post-ictal, ictal and post-ictal.The finding from the t-test implies that the brain activity consistently shows no change between the pre-ictal and post-ictal phase for all 5 features, except for the Min feature of Caudate $(p=0.01)$. Statistically significant changes with $p$ value $<0.05$ were found in lefthippocampus (in Mean and Standard deviation feature),right hippocampus (Max),left accumbens (Power spectral density), right accumbens (Mean, Max, Standard deviation and Power spectral density ), and left amygdala (Min) during the ictal phase.

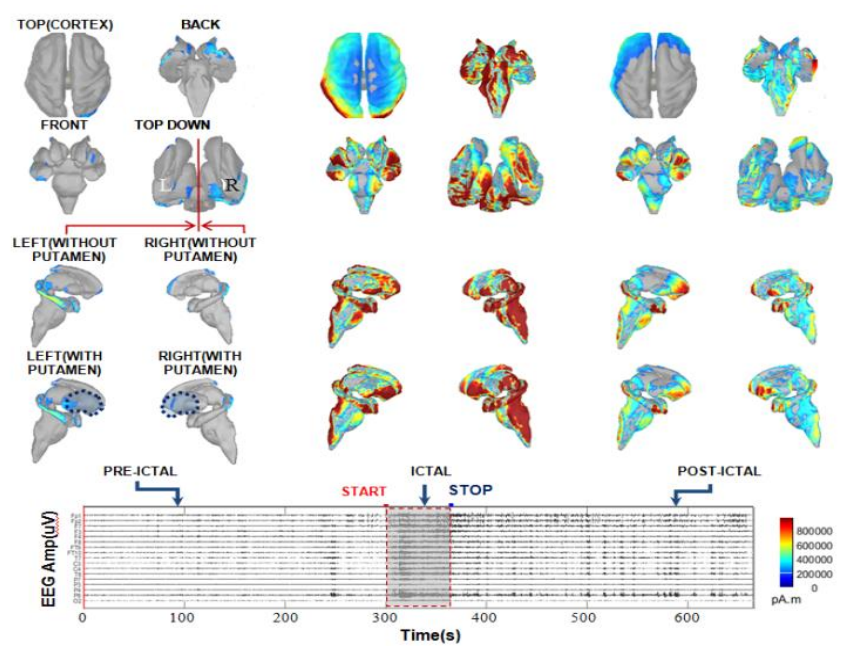

Fig.2 Alternations in a deep brain activity during pre-ictal, ictal to postictal. The 3-D brain structures from different views are constructed using the electrical energy from the electrophysiological. The colors represent the energy of particular brain areas in a certain time. The bottom graph is the EEG data of an individual seizure episode with 17 monopolar channels, containing $5 \mathrm{~min}$ of apre-ictal period, ictal period, and $5 \mathrm{~min}$ of apost-ictal period. A gray shaded window indicates the seizure episode.

Generally, our preliminary resultshows that there are increasing activities in the subcortical structures during the generalized epilepsy including the left and right hippocampus, left and right accumbens, and left amygdala. It is also found that the affected brain regions are quite symmetrical except for the amygdala which only shows significantly on the left side. It can be explained that since the Amygdala (L) region is defined by the Min feature, the time series may not be a reliable feature.The Min feature also determines an absurd result that there is a significant 
difference between pre-ictal and post-ictal in Caudate (L). Our findings are closely related to the other works using different types of measures.

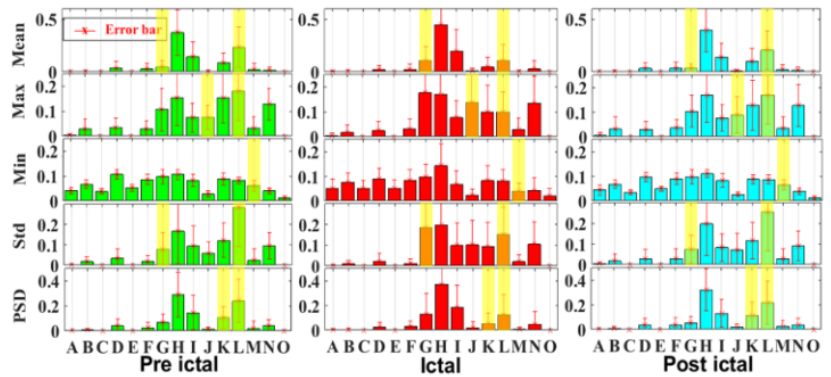

Fig. 3 Histogram of 5 features including mean, max, min, standard deviation, and power spectral density over three consecutive pre-ictal, ictal and post-ictal periods. The brain regions under the consideration inludesA: Thalamus Right, B: ThalamusLeft, C: Pallidum Right, D: Caudate Left, E: PallidumLeft, F: Caudate Right,G: Hippocampus Left, H: Putamen Left, I: Putamen Right, J: Hippocampus Right, K:Accumbens Left, L: Accumbens Right, M: Amygdala Left, N:Amygdala Right, and O: Brainstem. The areas are in the order of distance to the reference point. defined as the location of the intermediate mass of the thalamus (structure connects 2 sides of thalamus). The features at the Hippocampus Left and Right, Accumbens Left and Right, and Amygdala Left with the most significant changes are highlighted.

The study of the distribution of (14C)2-deoxyglucose (2-DG) in the generalized epilepsy rat brain (induced by stimulation of a kindled amygdala) also shows an expanded neural network with strong bilateral 2-DG auto radiographic activity in the anterior neocortex, striatum and thalamus[16].The electrophysiological model presented in this study supports the idea that not all regions of the whole brain are affected equally by generalized epilepsy. It is also notable with the result that the thalamus, caudate, pallidum, putamen and brainstem region are hardly affected. In short, findings in this study suggest that generalized epilepsy may not homogeneously affect the entire brain, but rather only involve in some specific regions.

\section{Conclusions}

Generalized epilepsy is a subgroup of epilepsy that required the utmost medical attention. However, we hardly acknowledge the behaviour, the onset, and the mechanic of this particular epilepsy. A better understanding of generalized epilepsy may be derived from the investigation of subcortical activity. The role of these structures can be unveiled by medical imaging techniques or by computational models. The surface EEG is a bright candidate since it is not only high resolution, easy to develop a medical device for patient management, but also it is possible to detect abnormalities in the inner brain structures if given a judicious tool. Our research focuses on how to use the electrophysiological model that involves in the surface EEG signal and the anatomical features to analyze the attributes and dynamic of a deep brain activity during the ictal stage of generalized epilepsy. The preliminary result shows that electrophysiological activities are uniformly distributed over the brain areas during the epileptic seizures. There are notable changes in energy in some deep brain structures such as hippocampus, accumbens, amygdala.

We are complementing the findings from this study with the graph theory and EEG/fNIRS coupling. The invivo experiments are going to be done to investigate the clinical potential of the EEG signals as a biomarker for the tracking of the epilepsy onset, progress, and recovery. The proposed EEG-driven model can be used to predict the abnormalities onset prior the structure changes, detect the abnormalities in deep brain structures with a higher sensitivity, and to facilitate the development of continuously monitoring epilepsy devices.

\section{ACKNOWLEDGMENT}

British Council-Newton Fund is acknowledged for their support.

\section{REFERENCES}

1. Banerjee P.N. et al. (2009) The descriptive epidemiology of epilepsy-a review. Epilepsy research, 85(1): 31-45

2. Annegers J.F. et al. SUDEP: Overview of definitions and review of incidence data. Seizure, 8(6): 347-352

3. De Tisi J. et al. (2011) The long-term outcome of adult epilepsy surgery, patterns of seizure remission, and relapse: a cohort study. Lancet, 378(9800): 1388-95

4. Scharfman H.E. et al. Structural and functional asymmetry in the normal and epileptic rat dentate gyrus. Journal of Comparative Neurology, 454(4):424-439

5. Norden A.D. et al. (2002) The role of subcortical structures in human epilepsy. Epilepsy \& Behavior, 2002. 3(3): 219-231.

6. Blumenfeld, H., The thalamus and seizures. Archives of neurology, 59(1): 135

7. Attal Y. et al. (2007) Modeling and detecting deep brain activity with MEG \& EEG. In Proceeding of Annual International Conference of the IEEE Engineering in Medicine and Biology Society, 2007, pp 937-40

8. Omata K. et al. (2013) Spontaneous slow fluctuation of EEG alpha rhythm reflects activity in deep-brain structures: a simultaneous EEG-fMRI study. PloS one, 8(6): e66869

9. Lainscsek C. et al. Non-Linear Dynamical Analysis of EEG Time Series Distinguishes Patients with Parkinson's Disease from Healthy Individuals. Frontiers in Neurology, 4

10. Wendling F. et al. (2005) Interictal to ictal transition in human temporal lobe epilepsy: insights from a computational model of intracerebral EEG. Journal of Clinical Neurophysiology, 22(5): 343356

11. Wendling F. et al. (2001) Interpretation of interdependencies in epileptic signals using a macroscopic physiological model of the EEG. Clinical Neurophysiology, 112(7): 1201-1218

12. Tadel F. et al. (2011) Brainstorm: A User-Friendly Application for MEG/EEG Analysis. Computational Intelligence and Neuroscience, 13.

13. Gramfort A. et al. OpenMEEG: opensource software for quasistatic bioelectromagnetics. BioMedical Engineering OnLine, 9(1): 1-20.

14. Mosher J.C. et al. (1993) Error bounds for EEG and MEG dipole source localization. Electroencephalography and Clinical Neurophysiology, 86(5): 303-321

15. Lin F.-H. et al. (2006) Assessing and improving the spatial accuracy in MEG source localization by depth-weighted minimum-norm estimates. NeuroImage, 31(1): 160-171

16. McIntyre D.C. et al. (1991) Distribution of [14C]2-deoxyglucose after various forms and durations of status epilepticus induced by stimulation of a kindled amygdala focus in rats. Epilepsy Res, 10(23): $119-33$

Author: Trung Le

Institute: International University- Vietnam National University

Address: Quarter 6, Linh Trung, Thu Duc District

City: Ho Chi Minh

Country: Vietnam

Email: trung.le@hcmiu.edu.vn 